Article

\title{
On the Achievable Information Rate of Probabilistic Shaping QAM Order and Source Entropy in Visible Light Communication Systems
}

\author{
Peng Zou, Fangchen Hu, Yiheng Zhao and Nan Chi * \\ Department of Information Science, Fudan University, Shanghai 200433, China; \\ 18110720058@fudan.edu.cn (P.Z.); 18110720018@fudan.edu.cn (F.H.); zyh@fudan.edu.cn (Y.Z.) \\ * Correspondence: nanchi@fudan.edu.cn
}

Received: 27 May 2020; Accepted: 20 June 2020; Published: 23 June 2020

check for updates

\begin{abstract}
Probabilistic shaping (PS) is a powerful tool that can realize a flexible data rate and high spectrum efficiency. However, the performance of PS is closely related to the quadrature amplitude modulation (QAM) order, source entropy (SE), normalized generalized mutual information (NGMI), and achievable information rate (AIR). In this paper, we investigated the relationship between PS QAM order, SE, NGMI, and AIR performance for the first time in the visible light communication (VLC) system. Under a specific signal-to-noise ratio (SNR), the PS QAM order and SE in this paper can realize a high AIR at a preset NGMI threshold with the lowest computation complexity, which is just 0.1 bit smaller than the highest AIR. Simulation and experimental results show that the NGMI fluctuates between only \pm 0.005 of the preset NGMI threshold, which proves that the scheme proposed in this paper is feasible.
\end{abstract}

Keywords: probabilistic shaping; visible light communication; achievable information rate; normalized generalized mutual information

\section{Introduction}

Visible light communication (VLC) is a promising candidate for future wireless communication [1,2]. By utilizing the LED as the transmitting end and the PIN as the receiving end, VLC can be low-cost and can realize no electromagnetic interference communication without spectrum authorization [3]. In recent years, the demand for VLC traffic is continuously increasing. Advanced modulation formats, such as carrier-less amplitude phase (CAP) and orthogonal frequency division multiplexing (OFDM), combined with quadrature amplitude modulation (QAM), are widely used in VLC to increase the capacity of VLC systems [4-6]. However, traditional QAM has two apparent disadvantages: First, traditional QAM has a coarse granularity in spectral efficiency and cannot make full use of the channel capacity [7]. Second, traditional QAM is not the optimal constellation for the channel, which has an apparent gap to the Shannon capacity [8]. Therefore, it is necessary to optimize the transmitted signal to make it more suitable for the distribution of the channel.

In recent years, probabilistic shaping (PS) has become a hotspot in high data rate transmission $[9,10]$. By changing the probability of each constellation point to make a Gaussian-like constellation, PS could provide additional shaping gain to eliminate the gap between the Shannon capacity and system source entropy (SE). It is proven that in the additive white noise Gaussian (AWGN) channel, the Maxwell-Boltzmann distribution is the optimal probability distribution for the standard QAM constellation, which could provide shaping gain of at most $1.53 \mathrm{~dB}$ [11]. Furthermore, PS can realize arbitrary SE and rate adaption transmission according to channel state information [12]. Among the ways to realize a PS system, probabilistic amplitude shaping (PAS) is one of the more popular 
methods $[13,14]$. At the transmitting side, the PAS system utilizes constant composition distribution matching (CCDM) and low-density parity-check (LDPC) codes to implement binary to non-uniform symbol mapping $[15,16]$. At the receiver side, inverse CCDM and bit-metric decoding is implemented to recover the non-uniform symbol to binary streams and improve the system performance [17]. As end-to-end binary decoding will introduce an additional computation complexity and latency to the system, generalized mutual information (GMI) and normalized GMI (NGMI) are introduced to measure the system performance more conveniently [18]. In [19], the author has proven that the NGMI is a powerful tool in predicting the bit error rate (BER) of the post forward error correction (FEC) after soft decision bit-metric decoding. Therefore, it is convincible to utilize the GMI and NGMI as criteria. Additionally, the achievable information rate (AIR, also named as net transmission rate) could give out the actual system information rate after removing coding redundancy. It is proven to be a fairer way for different PS constellations and source entropies [20,21].

The advantages of PS have attracted many scholars to investigate the superior performance in different scenarios. It has been proven that PS is superior to the uniform constellation and geometrical shaping. In [22], PS constellations with static entropies could provide more than $1 \mathrm{~dB}$ signal-to-noise ratio (SNR) sensitivity gain for 256QAM in the AWGN fiber system. In [12,21] and [23], the PS constellation could realize $15.5-40 \%$ more transmission reach in optical systems. However, these papers operate at a fixed QAM order and pay little attention to the QAM order and its corresponding $\mathrm{SE}$, according to different channel state information, which is a fundamental issue to realizing higher system performance. In [24], the author concludes that a 4-probability mass function (PMF) is sufficient for 64QAM when a mismatch of $0.1 \mathrm{~dB}$ SNR is acceptable. However, the shaping factor of PMF is optimized by maximizing the system's mutual information (MI), which cannot reflect the system AIR without coding redundancy. Furthermore, the VLC system is different from the fiber system and the performance of the PS needs to be verified. The optimal SE of PS-256QAM is investigated in the entropy loading discrete multi-tone system [25]. This study is carried out in a multi-carrier modulation system, which will inevitably suffer from high peak to average power ratio (PAPR). Additionally, the optimal QAM order of PS at different SNR is not revealed. In the VLC system, there is little research on the selection of the PS QAM order and SE, which can meet the specific NGMI threshold with high AIR.

In this paper, we proposed a novel PS QAM order and SE selection scheme that can realize a high AIR at the lowest computation complexity, and meet the NGMI threshold. According to the channel SNR and the proposed schemes, the SE can be adjusted to meet the NGMI threshold. The AIR loss to the highest AIR is approximately 0.1 bpQs, while the computation complexity is the lowest among other possible combinations of SE and QAM order. The simulation results show that the NGMI of the PS signal fluctuates only around the threshold of \pm 0.004 . Experimental results proved that by just subtracting $0.1 \mathrm{bpQs} \mathrm{SE}$, the system can work above the NGMI threshold at all the $\mathrm{V}_{\mathrm{pp}}$, and the fluctuation range of the experimental result is only between the threshold of \pm 0.005 .

The following sections are organized as follows: the principle of the GMI, NGMI, and signal distribution in this paper are shown in Section 2. In Section 3, the experimental setup is introduced. Then, the system performance from PS QAM order 32 to 512 is measured in Section 4. In Section 5, we will draw the conclusion.

\section{Principles of Probabilistic Shaping}

The famous Shannon capacity of the channel can be expressed as:

$$
C=\log _{2}(1+S N R) .
$$

The SNR is measured by the uniform power training sequence. It has been proven that for the QAM constellations, the optimal Gaussian source can be generated by making the constellation yield the Maxwell-Boltzmann distribution [11]. If we assume the M QAM constellations are taken from a set 
with complex values $\chi=\left\{x_{0}, x_{1}, \ldots, x_{M-1}\right\}$, the Maxwell-Boltzmann (MB) distribution for PS M-QAM is expressed as:

$$
P\left(x_{i}\right)=\frac{1}{\sum_{j=0}^{M-1} e^{-\lambda\left|x_{j}\right|^{2}}} e^{-\lambda\left|x_{i}\right|^{2}}
$$

The $\lambda$ is the shaping factor of the PS signal. The larger the $\lambda$ is, the greater the difference in probability between the inner and outer circles of the constellation points. In this paper, we utilize the famous probabilistic amplitude shaping (PAS) system with the MB distribution to realize the PS scheme. For more details of PAS, please refer to the article [14]. As the GMI and NGMI can adequately reflect the decoding performance of soft decision LDPC (SD-LDPC) in the PAS system, we utilize them as the metrics in this study. The GMI can be expressed as follows:

$$
G M I \approx \frac{1}{N} \sum_{k=0}^{N-1}\left[-\log _{2}\left(P_{k}\right)\right]-\frac{1}{N} \sum_{k=0}^{N-1} \sum_{i=1}^{M}\left[\log _{2}\left(1+e^{\left.(-1)^{b_{k, i}} \Lambda_{k, i}\right)}\right]\right.
$$

The $b_{k, i}$ are the transmitting bits of the $\mathrm{k}_{\mathrm{th}}$ symbol. $\Lambda_{k, i}$ is the soft bit-wise log-likelihood ratio (LLR) output which can be expressed as:

$$
\Lambda_{k, i}=\log \frac{\sum_{x \in \chi_{1}^{i}} q_{Y \mid X}\left(y_{k} \mid x\right) P(x)}{\sum_{x \in \chi_{0}^{i}} q_{Y \mid X}\left(y_{k} \mid x\right) P(x)}
$$

The $\chi_{1}^{i}$ and $\chi_{0}^{i}$ represent the subsets in $\chi$ whose $\mathrm{i}_{\text {th }}$ bit is 1 or $0 . q_{Y \mid X}\left(y_{k} \mid x\right)$ is the channel probability mass function (PMF). For the additive white Gaussian noise (AWGN) auxiliary channel with bit interleaved coded modulation (BICM) and a noise variance of $\sigma^{2}$, the PMF can be expressed as:

$$
q_{Y \mid X}\left(y_{k} \mid x\right)=\frac{1}{\sqrt{2 \pi \sigma^{2}}} e^{-\frac{\left|y_{k}-x\right|^{2}}{2 \sigma^{2}}}
$$

The normalized GMI (NGMI) reflects the ideal binary FEC rate of the system for error-free transmission. For M QAM PAS with an LDPC encoding procedure, the NGMI can be expressed as:

$$
N G M I=1-\frac{H-G M I}{\log _{2} M}
$$

Here, $H$ is the SE of M QAM, which is written as:

$$
H=\sum_{i=0}^{M-1}-P\left(x_{i}\right) \cdot \log _{2}\left(P\left(x_{i}\right)\right)
$$

Although the NGMI represents the ideal maximum rate, which can realize error-free decoding, it is practical to build a gap between the NGMI threshold and the code rate. In this paper, we utilize the NGMI threshold of 0.86 for the FEC code rate 5/6, and 0.92 for the FEC code rate $9 / 10$, to carry out our simulation and experiment [19]. If we define the FEC code rate as $R_{c}$, the NGMI is above the corresponding threshold, the achievable information rate (AIR) of PAS can be expressed as:

$$
A I R=H-\left(1-R_{c}\right) \cdot \log _{2} M .
$$

In this paper, we compare the simulation results of the NGMI for different PS QAM orders at the same AIR and preset NGMI threshold. Furthermore, we also consider optimizing the FEC code rate for each PS constellation and SNR to achieve a higher AIR. The SE of each PS QAM order is increased by $0.1 \mathrm{bit} / \mathrm{symbol}$ to find the highest AIR (HAIR) of each PS constellation when $R_{c}=N G M I-0.02$. 
Although we cannot obtain the relationship between the arbitrary code rate and the corresponding NGMI threshold, according to the results in Table III in [19], we consider it reasonable to set the gap between the code rate and its corresponding NGMI threshold to 0.02 . Therefore, the HAIR can be written as:

$$
H A I R=H-(1-(N G M I-0.02)) \cdot \log _{2} M .
$$

\section{Experimental and Simulation Setup}

The PS-CAP VLC experimental setup is shown in Figure 1. Firstly, two data streams are loaded by the CCDM. After LDPC encoding and QAM mapping, the symbols are upsampled 20 times. Then the real part and imaginary part of the signal are filtered by the Nyquist filter pairs, respectively. Afterward, the two signals add up and become a single signal, and are converted from a digital signal to an analog signal by the arbitrary waveform generator (AWG). After the equalization of a T-bridge hardware equalizer (Eq.) and amplification of the electrical amplifier (EA), the signal is coupled with DC and converted from an electric signal to an optical signal by the blue LED. After transmission in a $1.2 \mathrm{~m}$ water tank, the optical signal is collected and transferred to the current signal by a PIN (s10784). Then the current signal is converted to a voltage signal by a trans-impedance amplifier (TIA). After being amplified by an electrical amplifier, the signal is collected by an oscilloscope and loaded by the CAP demodulation offline processing block. For more details of CAP, please refer to the article [3]. Subsequently, the matched filter pairs, which are the corresponding shaping filters of Nyquist filter pairs, filter and separate the received signal into in-phase and quadrature signals. Then the signal is compensated by an LMS equalizer with the taps number of 43. The taps number is optimized and will not change throughout the experiment. After QAM demodulation, LDPC decoding, and the decoding of Inverse CCDM, the signal is recovered to bitstream again. The detailed parameters of Figure 1 are summarized in Table 1.

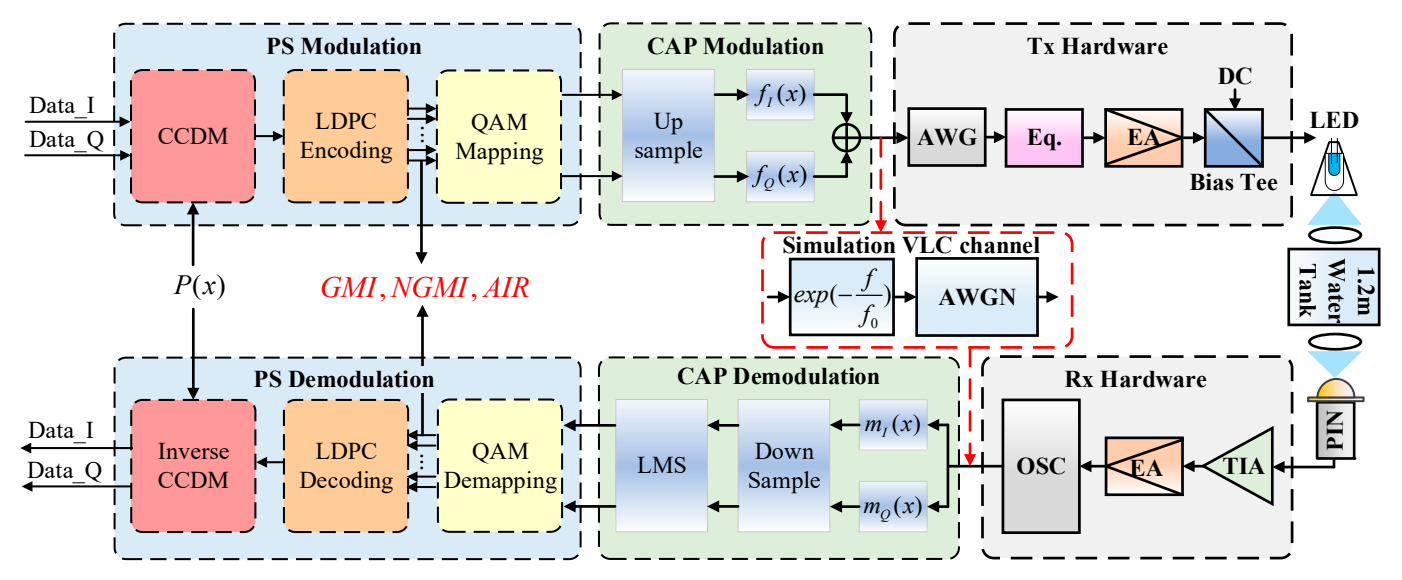

Figure 1. Experimental and simulation setup.

Table 1. Detailed parameters of experimental and simulation setup.

\begin{tabular}{cccc}
\hline \multicolumn{2}{c}{ Hardware Parameters } & \multicolumn{2}{c}{ Software Parameters } \\
\hline Terms & Parameters & Terms & Parameters \\
\hline AWG & AWG 710B & Roll-off of Nyquist filters & 0.205 \\
OSC & HP85545A & AWG sample rate & $400 \mathrm{MHz}$ \\
EA & ZHL-6A-S+ & OSC sample rate & $1 \mathrm{GHz}$ \\
Bias-Tee & ZFBT-4R2GW-FT+ & Upsample times & 20 \\
TIA & MAX3665 & LMS taps & 43 \\
LED & Special silicon LED & LDPC type & DVB-S2 \\
PIN & Hamamatsu S10784 & Simulation fading channel factor $f_{0}$ & 30 \\
\hline
\end{tabular}


For simulation, the PS modulation block, CAP modulation block, CAP demodulation block, and PS demodulation block remain unchanged, while the simulation VLC channel replaces the processing after CAP modulation and before CAP demodulation. The simulation VLC channel is the combination of an exponential fading channel in the frequency domain and additive white Gaussian noise (AWGN).

\section{Results and Discussion}

\subsection{Simulation Results}

The optimization problem in this paper is related to four variables: SE, SNR, NGMI, and QAM order. The simulation results in the AWGN channel are illustrated in Figure 2. Here we drew the surface plot of the SE, SNR, and NGMI of $64,128,256$, and 512 QAM. If we draw a plane with NGMI $=0.92$, then the intersection of the surface of this plane is the maximum SE of different SNRs that satisfy the threshold. In this figure, the NGMI rises with an increase in SNR for each SE. When the SNR is at a high level and the SE is at a low level, the NGMI saturates to 1, which means that the FEC code is not necessary for error-free decoding.
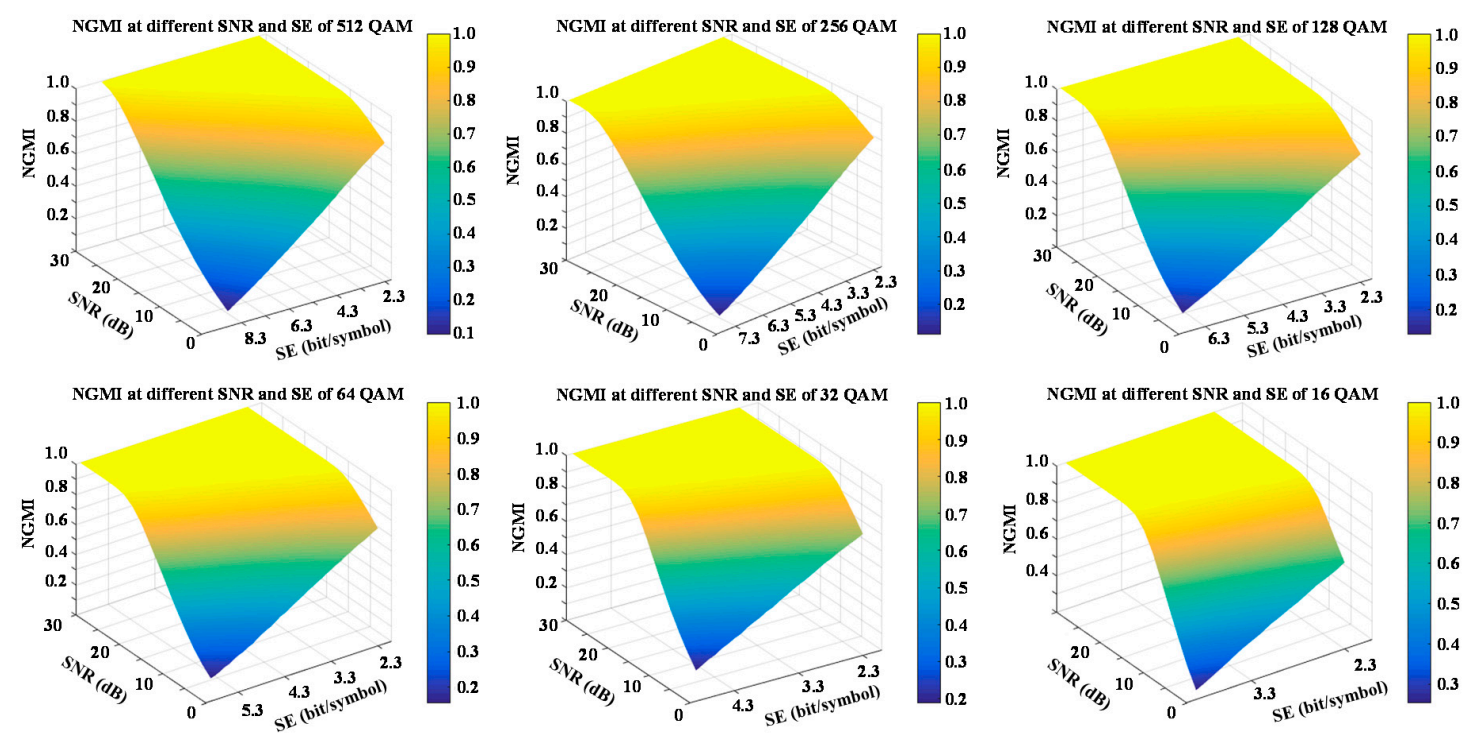

Figure 2. Relationship between normalized generalized mutual information (NGMI), source entropy (SE), and signal-to-noise ratio (SNR) with different probabilistic shaping (PS) QAM order of 16, 32, 64, 128,256 , and 512 .

In this study, we investigated the PS QAM order and SE in three scenarios:

(1) The NGMI threshold is 0.92 , which corresponds to the FEC code rate 9/10;

(2) The NGMI threshold is 0.86 , which corresponds to the FEC code rate $5 / 6$;

(3) The code rate $R_{c}=N G M I-0.02$.

In these scenarios, we try to find the QAM order and SE which own big enough AIR at each SNR with low computation complexity. The criteria are listed as follows: when the AIR gap between the alternative QAM orders is less than 0.1 bits per QAM symbol (bpQs), we choose the lower QAM order, which owns a much lower computation complexity in the CCDM-based PAS system. The reason is listed as follows: take the $\mathrm{SE}=5.3 \mathrm{bpQs}$ as an example. The innermost and outermost probability ratio of PS-64QAM, PS-128QAM, PS-256QAM, and PS-512QAM is 107.23, $8.86 \times 10^{3}, 3.50 \times 10^{10}$, $4.99 \times 10^{17}$, respectively. The probability ratio of the innermost and outermost is proportional to the storage depth of the digital to analog and the computation complexity of CCDM [16,21]. Therefore, it is hard to realize a perfect PS constellation by the higher QAM order. Furthermore, according to 
Equation (8), the system AIR without redundancy is closely related to the QAM order and SE at a specific NGMI threshold. The higher the QAM order, the more redundant bits the FEC code introduces, thereby introducing a higher system delay. Therefore, it is more reasonable and practical to realize a PS constellation with the lowest possible QAM order.

First, the simulation results are carried out on the AWGN channel. The optimal AIR and SE that satisfy the 0.92 NGMI threshold at different SNR are shown in Figure 3a,b, respectively. As Figure 3a shows, higher order QAM can realize better AIR performance. However, as raising one order will inevitably increase the computation complexity significantly, it is not sensible to always take the highest order as the PS order. In Figure 3a, the AIR gap bigger than 0.1 bpQs between 64QAM and 128QAM first appears at the SNR of $16.67 \mathrm{~dB}$. Meanwhile, the AIR gap between 128QAM and 256QAM first appears at $18.79 \mathrm{~dB}$. As the AIR gap between 256QAM and 512QAM is below $0.1 \mathrm{bpQs}$, the 512QAM is not taken into consideration.
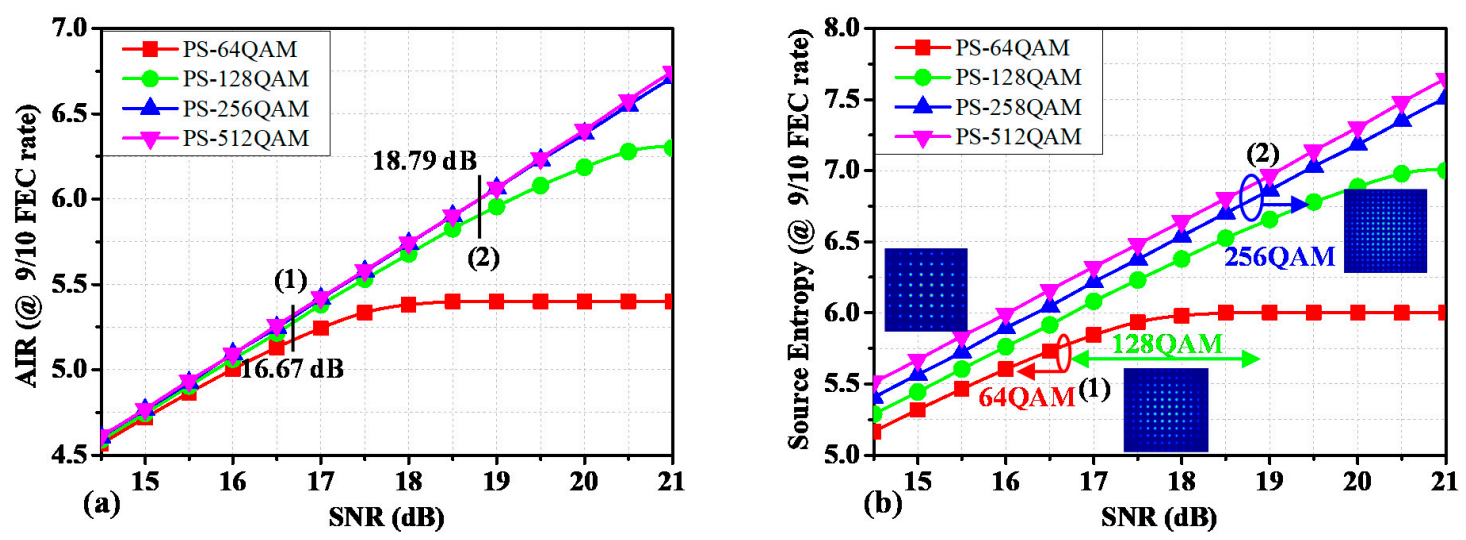

Figure 3. (a) AIR performance at 0.92 NGMI threshold; (b) SE performance at 0.92 NGMI threshold.

The optimal AIR and SE that satisfy the 0.86 NGMI threshold at different SNR are shown in Figure $4 \mathrm{a}, \mathrm{b}$, respectively. The transition SNR from QAM orders 6-7 and 7-8 are $15.65 \mathrm{~dB}$ and $17.63 \mathrm{~dB}$, respectively. As the FEC code rate decreases, higher SE is permitted to transmit more bits in the channel. Furthermore, the AIR performances of 256QAM and 512QAM are very close to each other. Similar to the results in Figure 3, it is not necessary to implement 512QAM in the simulation.
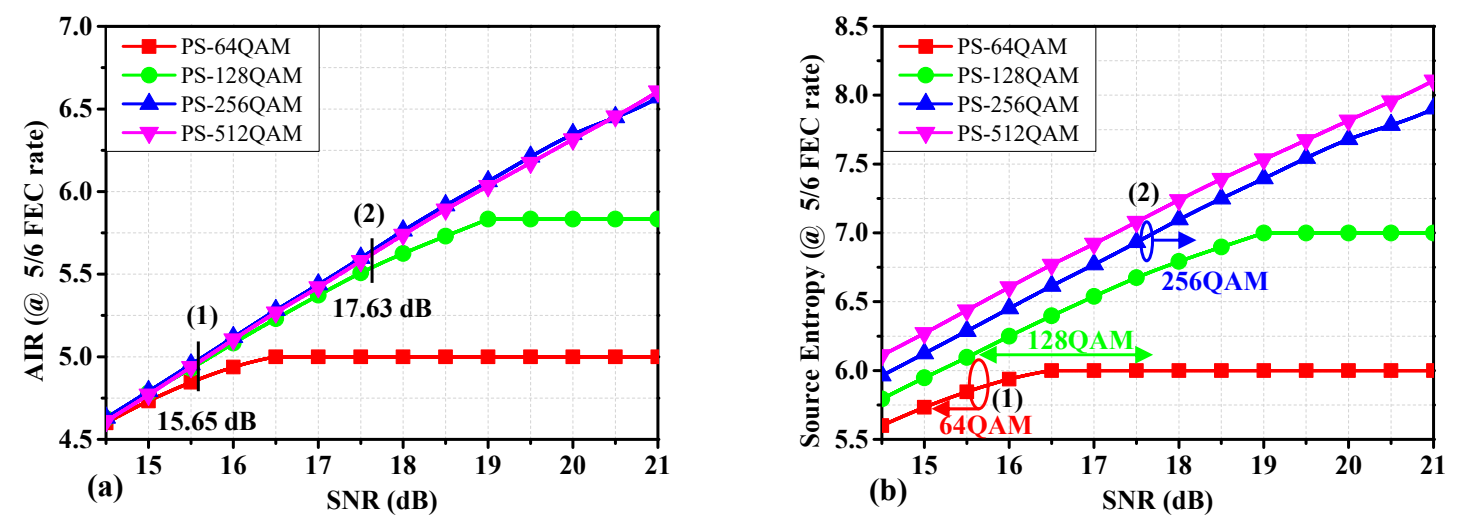

Figure 4. (a) AIR performance at 0.86 NGMI threshold. (b) SE performance at 0.86 NGMI threshold.

The optimal HAIR performance is shown in Figure 5a. The corresponding SE is shown in Figure 5b. As the target is to achieve the optimal AIR at the FEC code rate $R_{c}=N G M I-0.02$, we choose to change the SE by $0.1 \mathrm{bpQs}$ for each QAM order. This operation is different from scenario 1 and 2 because $R_{c}$ is a variable as well. The transfer point from 64 to 128QAM and 128 to 256QAM of SNR are $15.78 \mathrm{~dB}$ and $17.51 \mathrm{~dB}$, respectively. It is not necessary to implement $512 \mathrm{QAM}$ as well in this scenario. 

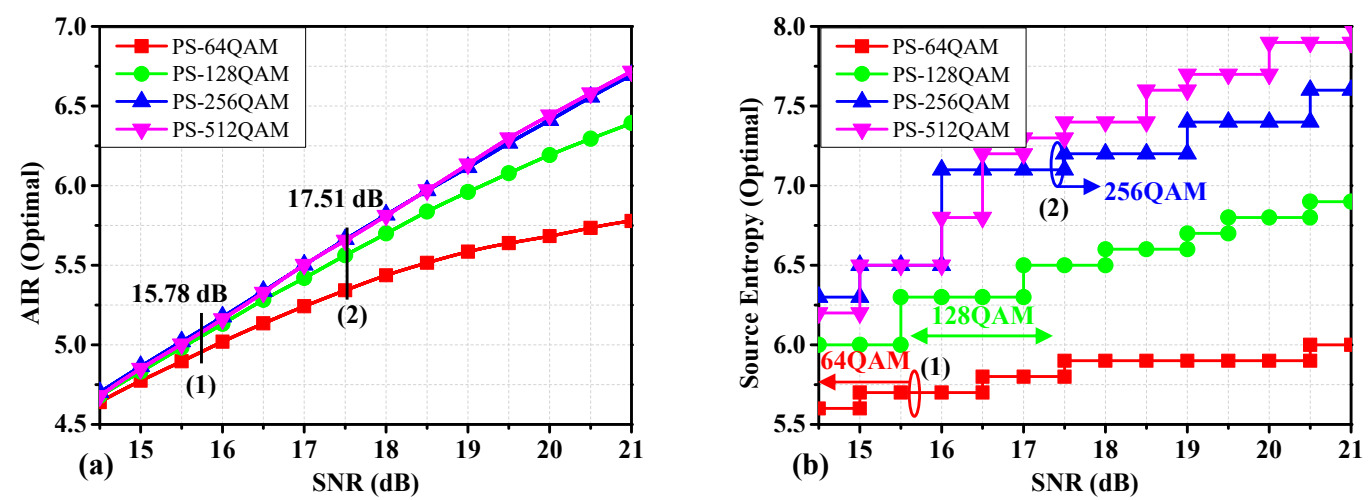

Figure 5. (a) HAIR performance. (b) Corresponding SE of HAIR.

For scenario 1-3, we can achieve the optimal SE at a specific SNR from Figures 3-5, respectively. Table 2 summarizes the QAM order exchanging point of scenario 1 to 3.

Table 2. QAM order exchanging point and corresponding SNR.

\begin{tabular}{ccccccc}
\hline \multirow{2}{*}{ Exchanging Points } & \multicolumn{2}{c}{ NGMI_th $=\mathbf{0 . 9 2}$} & \multicolumn{2}{c}{ NGMI_th $=\mathbf{0 . 8 6}$} & \multicolumn{2}{c}{ HAIR } \\
\cline { 2 - 7 } & $\begin{array}{c}\text { SNR } \\
\text { (dB) }\end{array}$ & $\begin{array}{c}\text { Shannon } \\
\text { Capacity }\end{array}$ & $\begin{array}{l}\text { SNR } \\
\text { (dB) }\end{array}$ & $\begin{array}{c}\text { Shannon } \\
\text { Capacity }\end{array}$ & $\begin{array}{l}\text { SNR } \\
\text { (dB) }\end{array}$ & $\begin{array}{c}\text { Shannon } \\
\text { Capacity }\end{array}$ \\
\hline$(1)$ & 16.67 & 5.57 & 15.65 & 5.24 & 15.78 & 5.28 \\
$(2)$ & 18.79 & 6.26 & 17.63 & 5.88 & 17.51 & 5.84 \\
\hline
\end{tabular}

Next, we simulate the performance of the CAP-based VLC system. First, the SNR performance in the CAP-based VLC system is measured. As the training sequence measures the channel SNR, it is crucial to know the relationship between the training sequence measured SNR and the PS signal SNR. We choose uniform 64QAM as the training sequence and measure the received SNR of the PS signal. Figure 6 shows the relationship between the PS signal SNR (SNR_PS) and the training sequence SNR (SNR_TS). The SE and QAM order of PS are chosen according to Figure 2b. We can see that the SNR of the PS signal matches well with that of the training sequence. The maximum gap between SNR_PS and SNR_TS is only $0.04 \mathrm{~dB}$. Therefore, it is reasonable to set the SE according to the training sequence SNR.

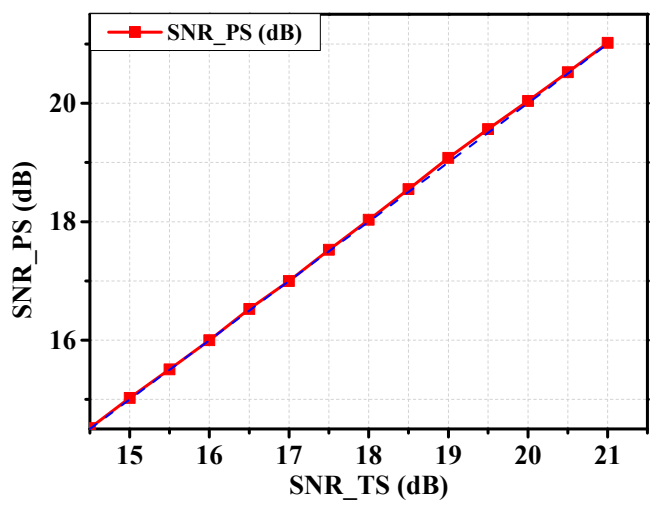

Figure 6. The PS signal SNR (SNR_PS) and training sequence SNR (SNR_TS).

The NGMI and AIR performance are shown in Figure 7a,b, respectively. The simulation results illustrate that the NGMI of scenario 1 and 2 fluctuates in a small range around 0.92 and 0.86 , respectively, which indicates that the relationship between SE and SNR in Figures 3 and 4 is accurate. The fluctuation range of 0.92 is from 0.916 to 0.921 , while that of 0.86 is from 0.855 to 0.861 . For scenario 3, the NGMI is shown by green circles. The AIR is shown in Figure $7 \mathrm{~b}$, and the optimal AIR performance is achieved 
by scenario 3. As the code rate of each point is optimized, this result is reasonable. However, the cost is to change the FEC code rate at each operation point, which is almost impossible in a practical system. Therefore, for a functional system, the NGMI threshold and FEC code rate should be fixed. As the NGMI below the threshold cannot permit error-free decoding for the system, we choose to subtract 0.1 bit from the preset SE in the experiment to ensure error-free decoding.
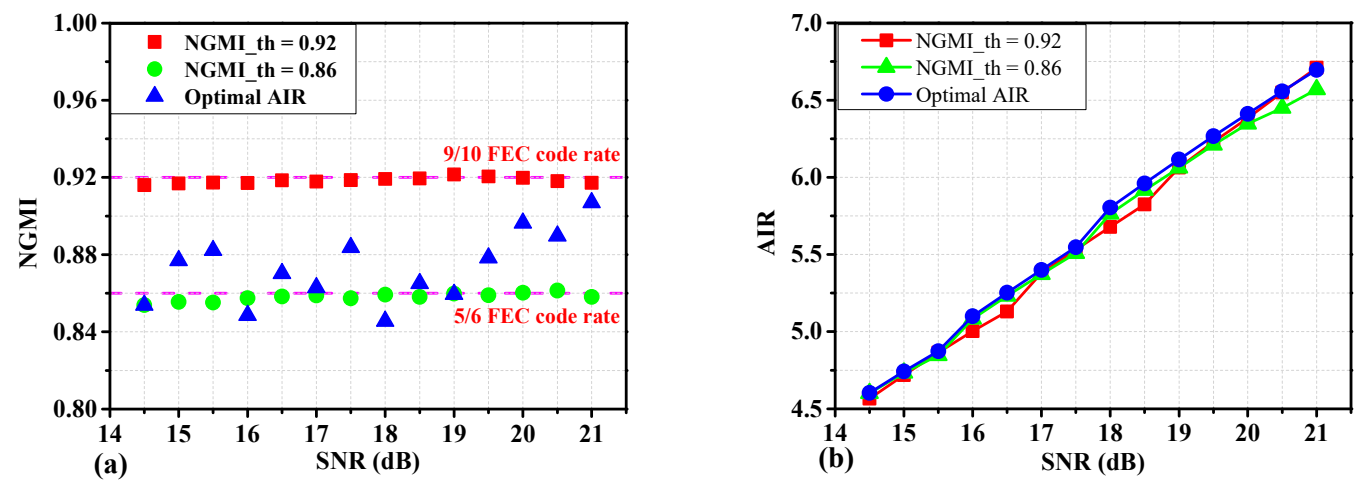

Figure 7. The NGMI and AIR simulation performance of scenario 1 to 3 .

\subsection{Experimental Results}

In Section 4.1, we verified the performance of our proposed PS scheme on the simulation platform. However, the channel of the experimental platform is much more complicated than that of the simulation platform. Therefore, it is necessary to verify the performance of the proposed PS scheme on the VLC experimental setup. Since we need to adjust the code rate according to the measured SNR in scenario 3, which is impossible to achieve in the actual system, we only tested the performance under the two thresholds of 0.86 and 0.92 on the experimental setup. To ensure the system meets the NGMI threshold, we choose to subtract the achieved SE by 0.1 bpQs.

Firstly, we adjusted the signal peak to peak voltage $\left(\mathrm{V}_{\mathrm{pp}}\right)$ from $0.19 \mathrm{~V}$ to $0.35 \mathrm{~V}$, and the measured SNR of uniform 64QAM is between $14.5 \mathrm{~dB}$ to $21 \mathrm{~dB}$ and shown as the blue bars in Figure 8a. The source entropies at different operation points are achieved by the measured SNR and shown as the green bars in Figure 8a. Figure 8b demonstrates the NGMI and AIR performance from $0.19 \mathrm{~V}$ to $0.35 \mathrm{~V}$. The NGMI fluctuates between 0.860 and 0.865 . Therefore, the experimental results prove the validity of the simulation results. At the top of Figure $8 b$, the received PS constellations of each $V_{p p}$ are shown.
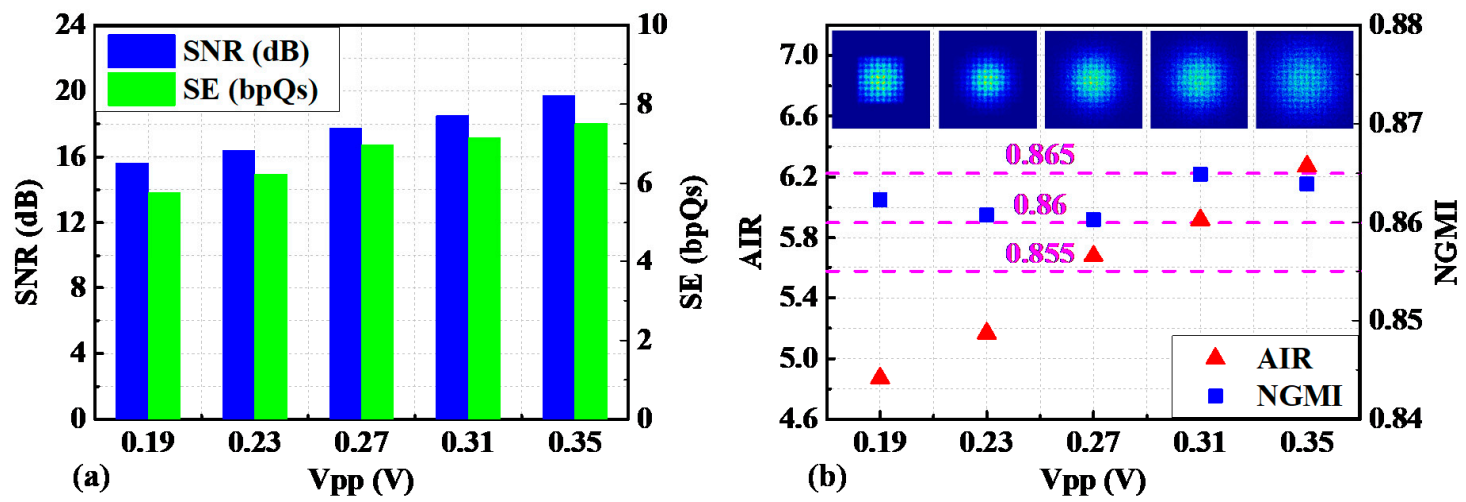

Figure 8. Experimental results at the NGMI threshold 0.86 .

Finally, we measured the system performance at the NGMI threshold 0.92. Similarly, the SNR of different operation points is adjusted between $14.5 \mathrm{~dB}$ and $21 \mathrm{~dB}$, and the source entropies are achieved according to the measured SNR in Figure 3a, which is shown in Figure 9a. The NGMI, GMI, and 
received PS constellations are shown in Figure $9 \mathrm{~b}$. The measured NGMI of PS constellations is all above the 0.92 NGMI threshold, and the maximum NGMI is 0.924 . The NGMI fluctuates between the \pm 0.005 of the preset NGMI threshold. Therefore, the simulation results match well with the experimental results, which proves that the PS mapping scheme is feasible.
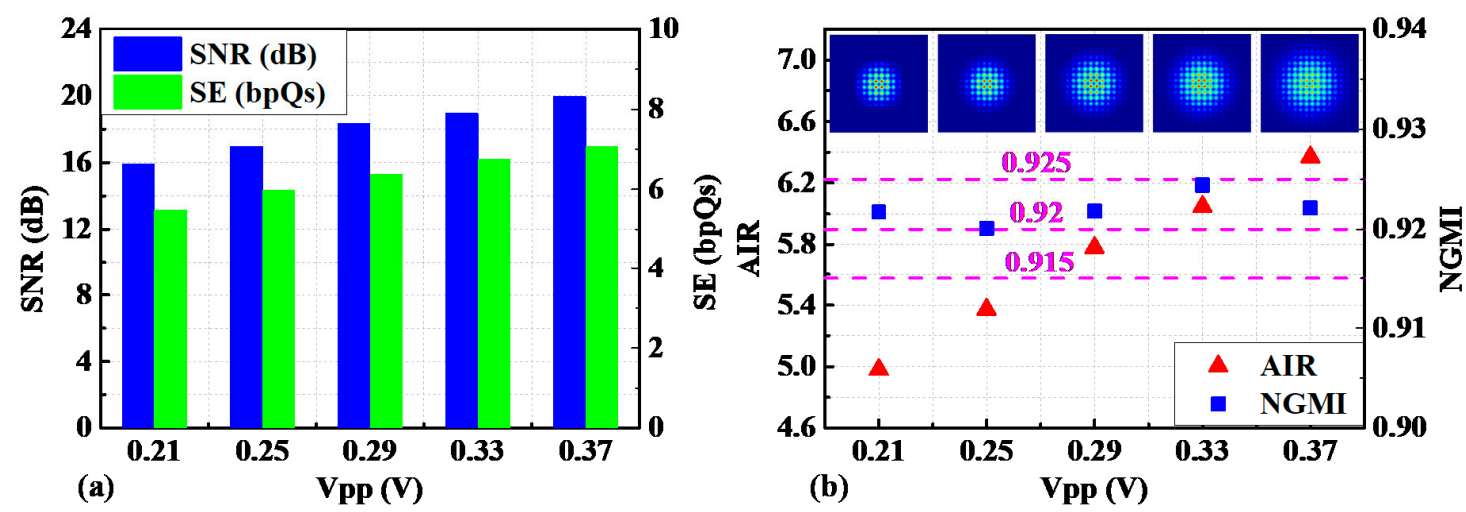

Figure 9. Experimental results at the NGMI threshold 0.92 .

\section{Conclusions}

In this paper, we investigated the relationship between the QAM order, SE, NGMI, and AIR in the PS-CAP VLC system for the first time. Simulation and experimental results demonstrate that with the aid of the proposed scheme in this paper, we can meet the NGMI threshold with the lowest computation complexity. Compared with the highest AIR, the AIR loss proposed in this paper is only 0.1 bpQs. Simulation results show that the NGMI only fluctuates within the range of \pm 0.004 around the preset NGMI threshold. Experimental results show that the NGMI are all above the preset NGMI threshold and only vary within a small scale by subtracting $0.1 \mathrm{bpQs}$ SE. Therefore, the scheme proposed in this paper is feasible for the AIR improvement of the PS signal. Additionally, with the scheme proposed in this paper, a very high AIR without coding redundancy can be achieved at the lowest cost according to the channel SNR, which only has a 0.1 bpQs gap at most, to the largest AIR of the system. Consequently, the scheme proposed in this paper has great potential in standardizing communication systems. In this study, the $V_{p p}$ is adjusted in a small range to suppress the nonlinear distortion. In the future, the AIR-based PS scheme in the nonlinear VLC system needs to be investigated. Besides, the performance of the proposed PS scheme needs to be verified in the multi-input multi-output (MIMO) VLC system as well.

Author Contributions: conceptualization, P.Z.; methodology, P.Z.; software, P.Z., and Y.Z.; validation, P.Z., and F.H.; formal analysis, P.Z., and F.H.; investigation, F.H., and Y.Z.; resources, F.H.; data curation, F.H., and Y.Z.; writing — original draft preparation, P.Z.; writing - review and editing, N.C.; visualization, F.H., and Y.Z.; supervision, Y.Z., and N.C.; project administration, N.C.; funding acquisition, N.C. All authors have read and agreed to the published version of the manuscript.

Funding: This research was funded by the National Key Research and Development Program of China (2017YFB0403603); National Natural Science Foundation of China (61925104).

Conflicts of Interest: The authors declare no conflict of interest. 


\section{References}

1. Jovicic, A.; Li, J.; Richardson, T. Visible light communication: Opportunities, challenges and the path to market. IEEE Commun. Mag. 2015, 51, 26-32. [CrossRef]

2. Chi, N.; Haas, H.; Kavehrad, M.; Little, T.; Huang, X. Visible light communications: Demand factors, benefits and opportunities. IEEE Wirel. Commun. 2015, 22, 5-7. [CrossRef]

3. Chi, N.; Zhou, Y.; Liang, S.; Wang, F.; Li, J.; Wang, Y. Enabling technologies for high-speed visible light communication employing CAP modulation. J. Lightware Technol. 2018, 36, 510-518. [CrossRef]

4. Shi, J.; Zhu, X.; Wang, F.; Zou, P.; Zhou, Y.; Liu, J.; Jiang, F.; Chi, N. Net data rate of 14.6 Gbit/s underwater VLC utilizing silicon substrate common-anode five primary colors LED. In Proceedings of the 2019 Optical Fiber Communications Conference and Exhibition, OFC 2019-Proceedings, San Diego, CA, USA, 3-7 March 2019; Volume 1, pp. 1-3.

5. Zhou, Y.; Zhu, X.; Hu, F.; Shi, J.; Wang, F.; Zou, P.; Liu, J.; Jiang, F.; Chi, N. Common-anode LED on a Si substrate for beyond $15 \mathrm{Gbit} / \mathrm{s}$ underwater visible light communication. Photonics Res. 2019, 7, 1019-1029. [CrossRef]

6. Wang, Y.; Chi, N. 8-Gb/s RGBY LED-based WDM VLC system employing high-order CAP modulation and hybrid post equalizer system employing high-order CAP modulation and hybrid post equalizer. IEEE Photonics J. 2015, 7, 1-7.

7. Xie, C.; Chen, Z.; Fu, S.; Liu, W.; He, Z.; Deng, L.; Tang, M.; Liu, D. Achievable information rate enhancement of visible light communication using probabilistically shaped OFDM modulation. Opt. Express 2018, 26, 367-375. [CrossRef] [PubMed]

8. Raphaeli, D.; Member, S.; Gurevitz, A. Constellation shaping for pragmatic turbo-coded modulation with high spectral efficiency. IEEE Trans. Commun. 2004, 52, 341-345. [CrossRef]

9. De Jauregui Ruiz, I.F.; Ghazisaeidi, A.; Sab, O.A.; Plantady, P.; Calsat, A.; Dubost, S.; Schmalen, L.; Letellier, V.; Renaudier, J. 25.4-Tb/s Transmission over transpacific distances using truncated probabilistically shaped PDM-64QAM. J. Lightwave Technol. 2018, 36, 1354-1361. [CrossRef]

10. Shi, J.; Zhang, J.; Li, X.; Chi, N.; Zhang, Y.; Zhang, Q.; Yu, J. Improved performance of high-order QAM OFDM based on probabilistically shaping in the datacom. In Proceedings of the 2018 Optical Fiber Communications Conference and Exposition (OFC), San Diego, CA, USA, 11-15 March 2018; pp. 1-3.

11. Kschischang, F.R.; Pasupathy, S. Optimal nonuniform signaling for gaussian channels. IEEE Trans. Inf. Theory 1993, 39, 913-929. [CrossRef]

12. Buchali, F.; Steiner, F.; Böcherer, G.; Schmalen, L.; Schulte, P.; Idler, W. Rate adaptation and reach increase by probabilistically shaped 64-QAM: An experimental demonstration. J. Lightwave Technol. 2016, 34, 1599-1609. [CrossRef]

13. Bocherer, G.; Steiner, F.; Schulte, P. Bandwidth efficient and rate-matched low-density parity-check coded modulation. IEEE Trans. Commun. 2015, 63, 4651-4665. [CrossRef]

14. Schmalen, L. Probabilistic constellation shaping: Challenges and opportunities for forward error correction. In Proceedings of the 2018 Optical Fiber Communications Conference and Exposition (OFC), San Diego, CA, USA, 11-15 March 2018; pp. 1-3.

15. Fehenberger, T.; Bocherer, G.; Alvarado, A.; Hanik, N. LDPC coded modulation with probabilistic shaping for optical fiber systems. Conf. Opt. Fiber Commun. Tech. Dig. Ser. 2015, 2015, 15-17.

16. Schulte, P.; Böcherer, G. Constant composition distribution matching. IEEE Trans. Inf. Theory 2016, 62, 430-434. [CrossRef]

17. Sheikh, A.; Graell I Amat, A.; Liva, G.; Steiner, F. Probabilistic amplitude shaping with hard decision decoding and staircase codes. J. Lightwave Technol. 2018, 36, 1689-1697. [CrossRef]

18. Cho, J.; Schmalen, L.; Winzer, P.J. Normalized generalized mutual information as a forward error correction threshold for probabilistically shaped QAM. In Proceedings of the 2017 European Conference on Optical Communication (ECOC), Gothenburg, Sweden, 17-21 September 2017; Volume 8, pp. 2-4.

19. Alvarado, A.; Agrell, E.; Lavery, D.; Maher, R.; Bayvel, P. Replacing the soft-decision FEC limit paradigm in the design of optical communication systems. J. Lightwave Technol. 2016, 34, 707-721. [CrossRef]

20. Vassilieva, O.; Kim, I.; Ikeuchi, T. On the fairness of the performance evaluation of probabilistically shaped qam. In Proceedings of the 45th European Conference on Optical Communication (ECOC 2019), Dublin, Ireland, 22-26 September 2019. 
21. Pilori, D.; Bertignono, L.; Nespola, A.; Forghieri, F.; Bosco, G. Comparison of probabilistically shaped 64QAM with lower cardinality uniform constellations in long-haul optical systems. J. Lightwave Technol. 2018, 36, 501-509. [CrossRef]

22. Fehenberger, T.; Alvarado, A.; Bocherer, G.; Hanik, N. On probabilistic shaping of quadrature amplitude modulation for the nonlinear fiber channel. J. Lightwave Technol. 2016, 34, 5063-5073. [CrossRef]

23. Buchali, F.; Bocherer, G.; Idler, W.; Schmalen, L.; Schulte, P.; Steiner, F. Experimental demonstration of capacity increase and rate-adaptation by probabilistically shaped 64-QAM. In Proceedings of the European Conference on Optical Communication (ECOC), Valencia, Spain, 27 September-1 October 2015; Volume 2015, pp. 2-4.

24. Fehenberger, T.; Lavery, D.; Maher, R.; Alvarado, A.; Bayvel, P.; Hanik, N. Sensitivity gains by mismatched probabilistic shaping for optical communication systems. IEEE Photonics Technol. Lett. 2016, 28, 786-789. [CrossRef]

25. Che, D.; Shieh, W. Achievable rate comparison between entropy and bit loading in a 100-Gb/s DM-DD DMT system. In Proceedings of the 2019 Optical Fiber Communications Conference and Exhibition (OFC), San Diego, CA, USA, 3-7 March 2019; Volume 1, pp. 7-9.

(C) 2020 by the authors. Licensee MDPI, Basel, Switzerland. This article is an open access article distributed under the terms and conditions of the Creative Commons Attribution (CC BY) license (http://creativecommons.org/licenses/by/4.0/). 